"Bubbly fl ow reconst ructi on usi ng the St ochast i c Est i mat i on wi th an el ectrode array"

\begin{tabular}{|l|l|}
\hline 著者 & $\begin{array}{l}\text { ZHOU Wei, SUZUKN Takao, N SH UM Toyohi ko, } \\
\text { YAMAMOTO Fuj i o }\end{array}$ \\
\hline $\begin{array}{l}\text { j our nal or } \\
\text { publ i cat i on ti t l e }\end{array}$ & Measur enent Sci ence and Technol ogy \\
\hline vol une & 19 \\
\hline number & 10 \\
\hline page range & 105402 \\
\hline year & 2008 10 \\
\hline URL & ht t p: //hdl . handl e. net /10098/2102 \\
\hline
\end{tabular}




\title{
Reconstruction of gas-liquid flows using the Stochastic Estimation with a 16-channel electrode array
}

\author{
"Bubbly flow reconstruction using the Stochastic Estimation with an \\ electrode array"
}

\section{W Zhou ${ }^{+}$, T Suzuki ${ }^{\#}$, T Nishiumi and F Yamamoto}

Graduate School of Engineering, University of Fukui, 3-9-1 Bunkyo, Fukui, Fukui, 910-8507, Japan

Email: zhouwei830409@gmail.com, takao@fv.mech.fukui-u.ac.jp,ni@u-fukui.ac.jp,yamamoto@fv.mech.fukui-u.ac.jp Current addresses:

+ Plant Engineering \& Energy Solution Headquarters, Hitachi Zosen Corporation, 1-7-89 Nanko-Kita, Suminoe-ku, Osaka, 559-8559, Japan

\# Acoustics \& Fluid Mechanics, Boeing Company, P.O.Box 3707, MC 67-ML, Seattle, WA 98124-2207, U.S.A.

PACS: 47.55.Ca, 47.54.De

\begin{abstract}
Bubble-injection methods are considered to be a prospective approach to reduce skin-frictional drag for ships. To apply this technique for unsteady flows, we attempt to introduce a feedback loop of air injection in future applications. Hence, we wish to establish reduced order models of unsteady bubbly flows from the information at the bottom of the ship, but this must be achieved with a limited number of sensors in practice. This motivates us to develop a reconstruction technique that recovers instantaneous phase information of gas-liquid flows. This study introduces the so-called "Stochastic Estimation" and reconstructs bubble distribution based on electrical impedance. We simulate the flow at the
\end{abstract}


bottom of a ship using a horizontal channel and install a 16-channel electrode array on the upper wall to acquire time-histories of electrical impedance. The relation between the bubble distribution and the electrical impedance is determined by simultaneous image measurement using a high-speed camera. Once the coefficients of the linear relation between the electrical impedance and the local void fraction are calculated, two-dimensional bubble distribution can be estimated only from voltages at the 16 electrodes. The proposed technique is examined over a range of void fractions and flow velocities, and the capabilities of the Stochastic Estimation applied to bubbly-flow reconstruction are demonstrated by comparing the reconstructed/predicted images with the original ones.

Keywords: bubbly flow, micro-bubble method, void fraction measurement, electrical impedance, stochastic estimation, flow reconstruction, flow control

\section{Introduction}

When large-scale ships sail, a major part of the drag is occupied by skin friction at the bottom of the ship hull. One of the prospective approaches to reduce the drag is a bubble-injection method, in which air bubbles with a diameter from tens of micrometers to several millimeters are injected into a boundary layer [1]. From previous studies [2-8], it is known under "steady" conditions that there exist ranges of void fractions and distances from the injector in which the skin friction is reduced. At very low void fractions (on the order of $\alpha \sim 0.01 \%$, $\alpha$ being the void fraction), the skin friction decreases with increasing void fraction, but it is effective only within a certain distance from the injector. At much higher void fractions $(\alpha>$ $5 \%$ ), on the other hand, the effect of drag reduction becomes rather greater downstream [8]. Outside these ranges, drag can increase depending on the distance from the injector and the bubble size, which may also be related to the flow velocity.

To effectively apply bubble-injection methods for unsteady conditions in future applications, we attempt to introduce a feedback loop, in which we must autonomously control the optimized air injection over time (research on flow control applied to marine vehicles is summarized in [9]). To establish a feedback control system, we typically 
introduce a "reduced order model" which represents instantaneous flow field (refer to, such as [10]). The requisites of reduced order models consist of: i) It characterizes key flow structures that govern the phenomenon to be controlled. ii) It projects an instantaneous field onto a set of a minimum number of parameters, preferably several coefficients with less computational cost. iii) It requires only a limited number of sensors to detect a flow quantity on time. For a bubble-injection control system, we may wish to represent bubble distribution at the bottom of the ship with a reduced order model, but it is difficult to capture instantaneous two-phase flow information with a limited number of sensors. Moreover, the measurement system must be implementable in the ship hull. Therefore, we must develop such a flow reconstruction technique when we introduce feedback control for bubble-injection methods.

The key of the reconstruction technique is the correlation between bubble distribution and a flow quantity measured by sensors. In previous studies, for example, the relations between the bubble passage and static pressure as well as shear stress have been reported [11, 12], yet the correlation with these quantities is weak to detect local phase information and impractical to reconstruct bubble distributions. To measure the distributions of void fractions with high resolution, radioactive or ultrasound techniques (as well as electrical impedance described below) [13-15] have been applied with tomography algorithms. In these techniques, however, substantial computational time is often required for iterations, and pairs of an emitter and a detector must be implemented in the line of sight; hence, these are undesirable for feedback control. Likewise, direct image measurement is not applicable because of its huge data information, which cannot be processed during limited time. For practical void-fraction measurement, electrical impedance has been widely introduced in applications to nuclear engineering, petroleum engineering as well as marine vehicles [14-19]. By directly implementing electrodes in the area of interests, the local phase information can be instantaneously acquired. The resolution can be enhanced by clustering electrodes, but unlike other applications [18], this must be achieved in a non-intrusive fashion for feedback flow control.

In our research, several attempts have been made to reconstruct two-phase flow images with minimum computational cost. In particular, efforts have been made to combine with 
image measurement, which compensates spatial resolution of the point-wise measurement in existing techniques. Pressure sensors [20] or shear-stress transducers [21] have been examined to reconstruct two-dimensional bubble distribution in a horizontal channel, and prospective results have been obtained with shear-stress transducers; however, we wish to exploit a better solution to improve our reconstruction techniques in terms of accuracy and resolution.

The objective of this study is to develop a technique that reconstructs unsteady bubble distribution based on electrical impedance combined with image measurement. We simulate flow at the bottom of a ship using a horizontal channel and inject bubbles whose diameters are on the order of a millimeter from a needle array. We then acquire time-histories of local electrical impedance from a 16-channel electrode array embedded on the upper wall of the channel and simultaneously capture two-phase flow images by a high-speed video camera from the bottom of the channel over time. From these data, we calculate linear coefficients relating the local phase information with the electrical impedance, and recover the projected bubble distribution using the Stochastic Estimation [22, 23]. With this technique, a two-dimensional bubble distribution is represented with a finite number of coefficients, and the limitation of resolution based on the point-wise measurement by electrodes is compensated by the image measurement. The proposed technique is examined over a range of void fractions and flow velocities, and the accuracy is evaluated based on the correlation between the reconstructed/predicted images and the original ones.

The outline of the paper is as follows: After the introduction, the experimental apparatuses and flow conditions are stated. Subsequently, the procedures of the data pre-processing including filtering and image processing are explained, followed by the algorithm description of the Stochastic Estimation. In the results and discussion, capabilities of the proposed technique are evaluated, and conclusions and implications are finally presented.

\section{Experimental apparatuses and flow conditions}

\subsection{Experimental apparatuses}


The experimental apparatuses are illustrated in figure 1. The rectangular horizontal channel is made of transparent acrylic resin with the cross-section of $10 \mathrm{~mm} \times 100 \mathrm{~mm}$ in height and width, respectively, and the length being $6000 \mathrm{~mm}$. Water is circulated through the system by a pump (TERADA, TFK-40), and the power of the pump is supplied with an inverter (MITUBISHI, FR-E520-1.5K, with an output frequency of $0.2 \sim 400 \mathrm{~Hz}$ ). The flow velocity is adjusted by the rotational speed of the pump controlled by the frequency of the inverter's input power. Bubbles with a diameter on the order of a millimeter are generated through a porous plate (approximately 2000 holes with the average diameter of $62 \mu \mathrm{m}$ within a $14 \mathrm{~mm} \times$ $48 \mathrm{~mm}$ area) by an air compressor (REXON, RAC-1530).

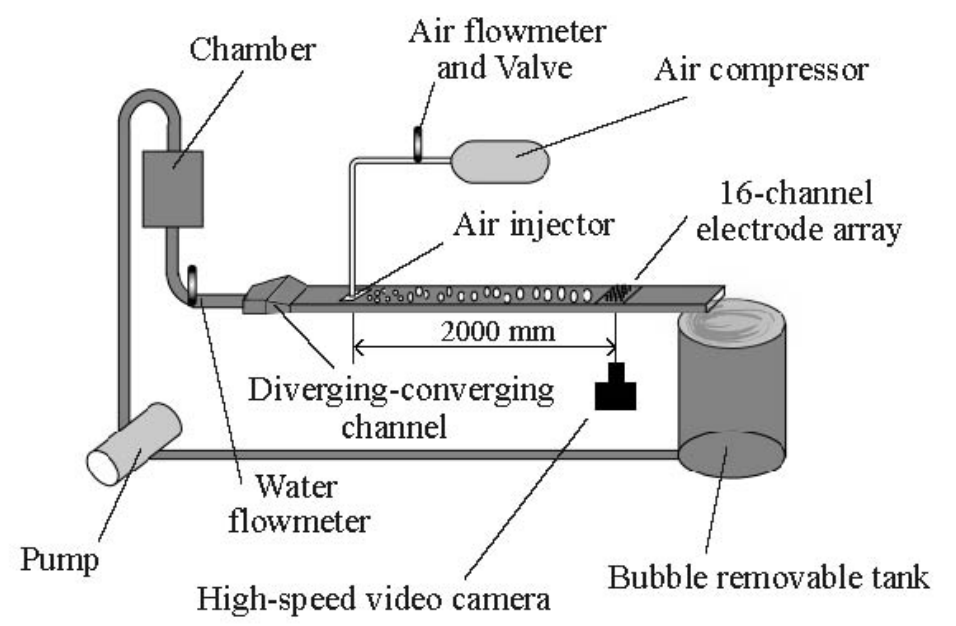

Figure 1. Schematic of the experimental apparatuses.

In the test section, an electrode array that consists of 16 electrodes $(4 \times 4)$ aligned in a 45 $\mathrm{mm} \times 45 \mathrm{~mm}$ square (i.e. the distance between adjacent electrodes is $15 \mathrm{~mm}$ ) is embedded on the upper wall of the channel $2000 \mathrm{~mm}$ downstream of the air injector. The photograph of the electrode array is displayed in figure 2.

For the electrodes' material, glassy carbon (whose product size measures $\phi 3 \mathrm{~mm} \times 10$ mm) available from the Tokai Carbon Co. Ltd. (GC-20SS,) is adopted because of the sufficient potential window and high durability. The diameter is chosen to be $3 \mathrm{~mm}$ so that the surface area is large enough to detect convecting bubbles. Each electrode is vertically penetrated into an acryl substrate base. They are then fixed tightly using epoxy resin and 
connected to lead wires using silver paste with high conductivity. The electrochemical system is illustrated in figure 3 .

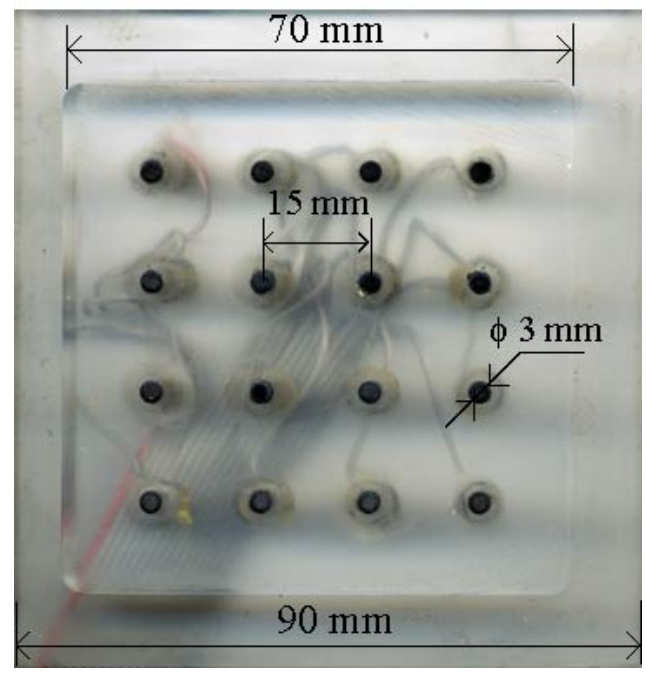

Figure 2. Photograph of the 16-channel electrode array.

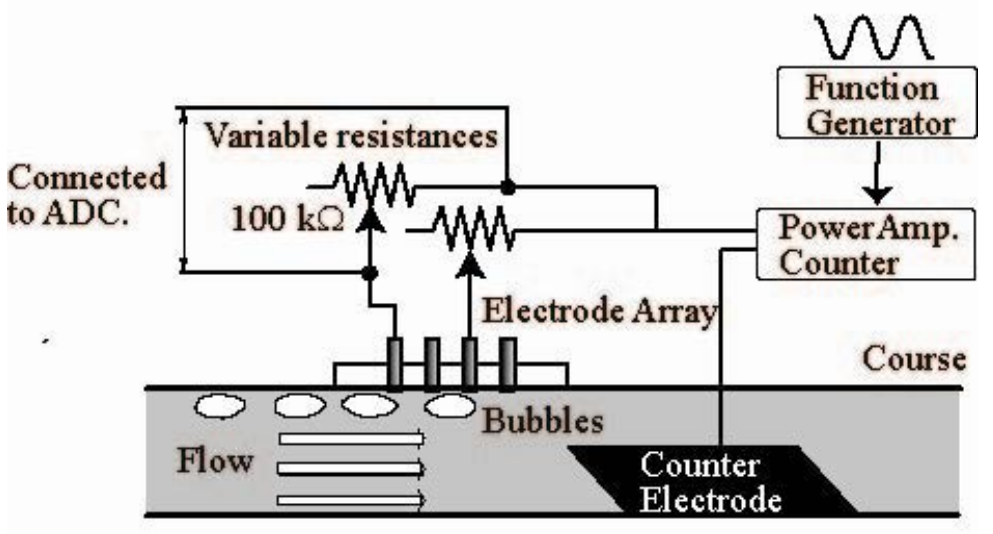

Figure 3. Schematic of the electrochemical system.

A stainless nickel plate in a square of $70 \mathrm{~mm} \times 70 \mathrm{~mm}$ is installed as a counter electrode at the bottom of the channel downstream. An alternating current function is generated by KENWOOD FG-272 and amplified by an audio power amplifier (SONY TA-N330ES). The output potential and function are calibrated using an oscilloscope (USB Instruments DS1M12 Stingray). The maximum potential of the output is fixed at $2.0 \mathrm{~V}$ considering the durability of the glassy carbon. When a bubble passes on the surface of the electrode, the resistance between each glassy carbon electrode and the counter electrode increases; consequently, some of the variable resistance values should decrease. The variation of the resistance largely 
depends on the surface phenomena; hence, it is convenient to capture bubbles locally convecting along the surface of the wall. We record these variations via $1 \mathrm{M} \Omega$ variable resistances fixed at about $100 \mathrm{k} \Omega$ for tap water using a 16-channel AD converter (CSI-360116 used as 16 bit, from Interface Co. Ltd.). The frequencies of the generated sine waves are $100 \mathrm{~Hz}$ and $1 \mathrm{kHz}$, and the sampling frequencies are 10 times the generated one (i.e. $1 \mathrm{kHz}$ and $10 \mathrm{kHz}$, respectively).

A CMOS high-speed video camera (Photron, FASTCAM-MAX 120KC) is focused approximately $5 \mathrm{~mm}$ beneath the upper wall of the channel at the center of the array. To create high contrast bubble images, the test section is illuminated by four metal halide lamps from the horizontal direction. From the electrode array, time-histories of electrical impedance are recorded, and simultaneously two-phase-flow images are taken by the camera from the bottom of the channel over time. The frame rate and the shutter speed of the camera are set to be $1000 \mathrm{fps}$ and $10^{-3} \mathrm{~s}$, respectively, and time-histories of voltages, corresponding to 2900 frames, are recorded for each flow condition.

\subsection{Experimental Conditions}

In this experiment, we compare the results among different flow velocities and void fractions. We define the void fraction as

$$
\alpha=\frac{\mathrm{Q}_{\mathrm{b}}}{\mathrm{Q}_{\mathrm{b}}+\mathrm{Q}_{\mathrm{w}}}
$$

where $\mathrm{Q}_{\mathrm{b}}$ and $\mathrm{Q}_{\mathrm{w}}$ denote the volumetric flow rates of bubble and water, respectively. Table 1 tabulates the experimental conditions of this study. We also list the Reynolds numbers based on the channel height $(10 \mathrm{~mm})$ for reference. Note that the uniformity of the bubble diameter is relatively high near the injector (its deviation is on the order of the bubble diameter or less at $250 \mathrm{~mm}$ from the injector [24]), but it becomes less uniform at the test window due to coalescence so that fully unsteady bubble distributions with relatively large diameters can be observed.

Although we specify the volumetric void fraction in this study, images taken by the high speed camera represent the projected bubble distribution. We also show in table 1 the relation between the volumetric void fraction and the projected void fraction, which is defined 
based on the area occupancy of the gas phase in the image. The results show that the projected void fraction is about $50 \sim 60 \%$ higher than the actual void fraction and nearly independent of the flow velocity.

Table 1. Experimental conditions and flow parameters.

\begin{tabular}{ccccc}
\hline & $\begin{array}{c}\text { Mean velocity } \\
U(\mathrm{~m} / \mathrm{s})\end{array}$ & $\begin{array}{c}\text { Volumetric void } \\
\text { fraction, } \alpha(\%)\end{array}$ & $\begin{array}{c}\text { Projected void } \\
\text { fraction }(\%)\end{array}$ & $\begin{array}{c}\text { Reynolds } \\
\text { Number }\end{array}$ \\
\hline Case A & 0.5 & 10 & 16 & 2490 \\
Case B & 0.5 & 15 & 25 & 2490 \\
Case C & 0.5 & 20 & 31 & 2490 \\
Case D & 0.75 & 20 & 32 & 3740 \\
Case E & 1.0 & 20 & 31 & 4980 \\
\hline
\end{tabular}

\section{Data pre-processing}

\subsection{Filtering of voltage data}

When bubbles cover or convect near the electrode, the electrical impedance sharply increases so that it captures the existence of bubbles. The corresponding voltage deficits appear in raw data recorded by the measuring system, but they are dominated by the sampling frequency of $1000 \mathrm{~Hz}$ (see figure 4). To apply the Stochastic Estimation, the envelope must be extracted from the raw data. Hence, the voltage profiles are filtered by the Fourier transform with two periods of the sampling frequency, and the resultant profile is overlaid in figure 4. Those filtered profiles are processed by the Stochastic Estimation.

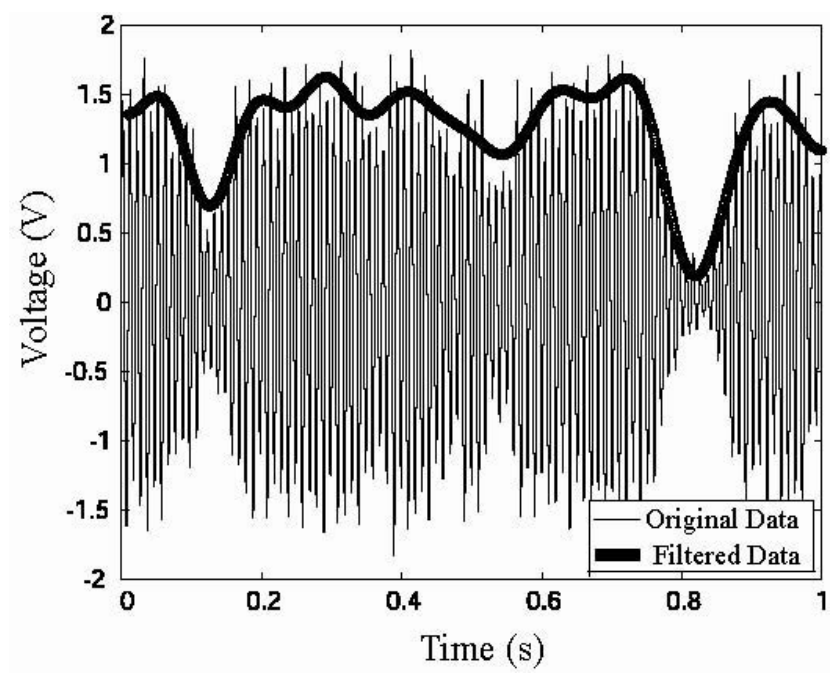

Figure 4. Typical voltage profile taken at an electrode. 


\subsection{Image pre-processing}

In this study, we record a series of grey-scale images with 256 levels by the high-speed camera (an example is shown in figure 5(a)); however, our reconstruction algorithm requires separating water and bubbles into white and black images, respectively. Therefore, the following image processing needs to be performed prior to the Stochastic Estimation.

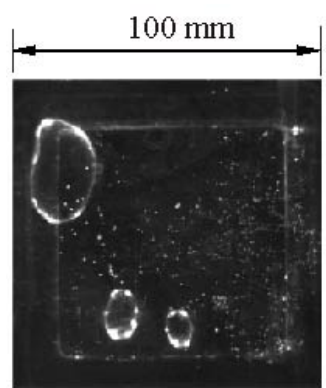

(a)

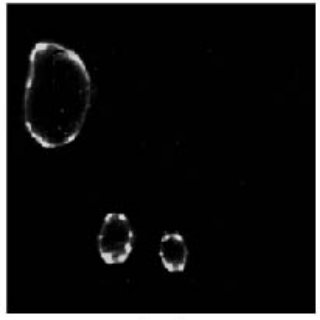

(c)

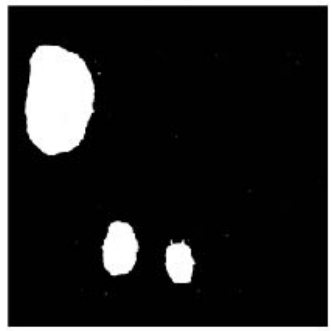

(e)

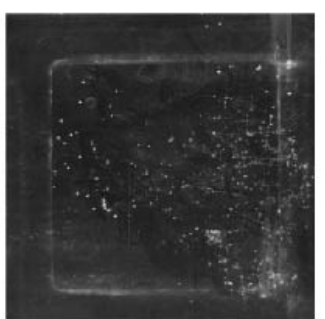

(b)

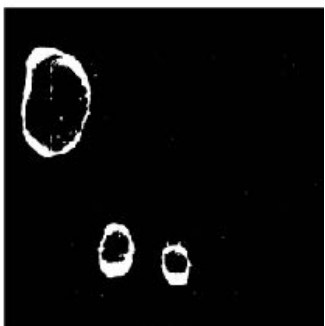

(d)

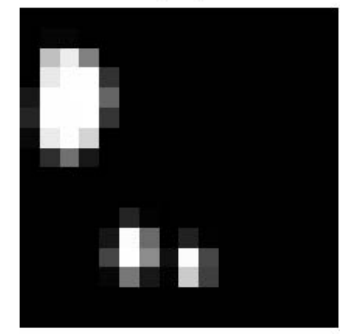

(f)

Figure 5. Example image at each step of pre-processing: (a) original image; (b) background;

(c) image without background; (d) binarized image; (e) filled image; (f) resultant image.

First, we eliminate the background image. Because almost only the boundaries of bubbles appear in the raw images and the number of bubbles is not too many, we can calculate the background by averaging all the images in the same condition. An example is shown in figure $5(\mathrm{~b})$. Subtracting the background image from the original ones, only the bubble images are extracted, as displayed in figure 5(c). Next, the aforementioned images 
are binarized, and the bubble regions are filled, as shown in figures 5(d) and (e), respectively. The resolution of original images is $1024 \times 1024$ pixels; however, in order to save computational cost for the reconstruction process, we reduce it to $16 \times 16$ grids and calculate the average brightness of each grid by averaging over $64 \times 64$ sub-pixels. The effect of the grid resolution will be briefly discussed in Section 5.2 later. Figure 5(f) exhibits a resultant image. In the results and discussion, we actually treat these pre-processed images as original ones and compare with the reconstructed/predicted images.

\section{Algorithm and quantification}

\subsection{Algorithm of the Stochastic Estimation}

We measure voltages at 16 points of the electrodes on the wall and represent them as $V_{1} \sim V_{16}$. Subsequently, we assume that the projected void fraction, $\alpha^{\prime}$, at each point can be approximated by the following linear combination:

$$
\alpha_{\mathrm{mn}}^{\prime}=C_{1_{m}} V_{1_{n}}+C_{2_{m}} V_{2_{n}}+\cdots+C_{16_{m}} V_{16_{n}},
$$

where the subscripts $m$ and $n$ represent the discretized spatial point on the image ( $m=1 \sim$ 256) and the quantity at time $t_{\mathrm{n}}(n=1 \sim N)$, respectively. To be precise, $\alpha_{\mathrm{m}}$ denotes the averaged brightness over the grid $m$ taken by the camera. We then define the following cost function to determine $C_{1} \sim C_{16}$ :

$$
J_{m}=\sum_{n=1}^{N}\left(\alpha_{m n}-\alpha_{m n}^{\prime}\right)^{2} .
$$

To minimize $J$, we differentiate equation (3) with respect to $C_{1} \sim C_{16}$ over $N$ sampling frames and obtain the following matrix equation:

$$
\left(\begin{array}{c}
C_{1 m} \\
C_{2 m} \\
\vdots \\
C_{16 m}
\end{array}\right)=\left(\begin{array}{ccc}
\sum_{1 n}^{N} V_{1 n}^{2} & \cdots & \sum_{1 n}^{N} V_{16 n} \\
\vdots & \ddots & \vdots \\
\sum_{n=1}^{N} Y_{16 n} V_{1 n} & \cdots & \sum_{n=1}^{N} Y_{16 n}^{2}
\end{array}\right)^{-1}\left(\begin{array}{c}
\sum_{n=1}^{N} \alpha_{m n} V_{1 n} \\
\vdots \\
\sum_{n=1}^{N} \alpha_{m n} V_{16 n}
\end{array}\right) .
$$

Once the coefficients, $C_{1} \sim C_{16}$, are determined from the equation above, the local brightness, $\alpha_{\mathrm{m}}$, at each point on the image can be simply estimated by summation from equation (2) at each frame. Thus, by storing a finite number of coefficients, we can 
reconstruct instantaneous bubble distributions with minimum computational cost using the Stochastic Estimation.

\subsection{Expression of the correlation}

We evaluate the results using a cross-correlation method. The following equation defines the correlation at time $t_{n}$ :

$$
R_{n}=\frac{\sum_{m=1}^{M}\left(f_{m n}-\bar{f}_{m}\right)\left(g_{m n}-\bar{g}_{m}\right)}{\sqrt{\sum_{m=1}^{M}\left(f_{m n}-\bar{f}_{m}\right)^{2} \sum_{m=1}^{M}\left(g_{m n}-\bar{g}_{m}\right)^{2}}},
$$

where $f_{m n}$ and $g_{m n}$ are the brightness values from an original image and the reconstructed one, respectively, using the Stochastic Estimation of grid $m$ at time $t_{n}$. The bars on the top denote the time average. We will specify the domain of summation for $m$ in Section 5.2. If the correlation is unity, the reconstructed image coincides with the original one.

While equation (5) denotes the correlation over the entire region, equation (6) below represents the time-averaged correlation at each grid:

$$
R_{m}=\frac{\sum_{n=1}^{N}\left(f_{m n}-\bar{f}_{m}\right)\left(g_{m n}-\bar{g}_{m}\right)}{\sqrt{\sum_{n=1}^{N}\left(f_{m n}-\bar{f}_{m}\right)^{2} \sum_{n=1}^{N}\left(g_{m n}-\bar{g}_{m}\right)^{2}}}
$$

We introduce this quantity to observe a detectable area of the electrode array. Furthermore, the correlation averaged both in space and time, i.e. $R \equiv \sum_{n=1}^{N} R_{n} \equiv \sum_{m=1}^{M} R_{m}$, is simply denoted by $R$ in the following sections.

\section{Results and discussion}

\subsection{Reconstructed and predicted images}

We separate our experimental results into two parts: the "reconstruction" part and the "prediction" part. As mentioned above, we calculate the coefficients, $C_{i}(i=1 \sim 16)$, based on $N$ sampling frames with the simultaneous image measurement, and redraw bubble 
distributions based on the voltage data with these coefficients. The redrawn images during the $N$ sampling frames are defined as "reconstruction," while those processed using the voltage data after the sampling period (i.e. without the image data) are referred to as "prediction." The reconstruction period corresponds to laboratory test data with simultaneous image measurement during pre-sailing, and the predicted period mimics a sailing condition when the feedback control is activated.

In the post-processing, we actually take intervals between the reconstruction and prediction parts based on the hypothesis that the coefficients determined in the reconstruction period can be used to estimate the bubble distribution during the prediction period. The first 1500 frames are used to determine the coefficients (i.e. the reconstruction period), and the last 1000 frames are evaluated for the prediction period after a 400 frame interval, unless otherwise stated.

To ensure the repeatability of the Stochastic Estimation, we run Case C $(U=0.5 \mathrm{~m} / \mathrm{s}$ and $\alpha=20 \%$, see table 1) on a different date and similarly perform the "reconstruction" process for two independent runs with 1000 frames. Compared with the result of the same condition discussed below, the deviation of these two additional runs is within $8 \%$ (increased) in terms of the averaged correlation.

Figure 6 displays examples of "reconstructed" images at (a) high and (b) low correlation frames in Case C. We also show the original images on the right for reference. When relatively large bubbles are convecting in the test section, the correlation is generally high. In contrast, it tends to drop when no or small bubbles are convecting. Figure 7 similarly exhibits examples of "predicted" images for the same conditions (Case C). Although the correlation becomes lower than that of the reconstructed cases, the agreement is still satisfactory in the middle of the domain.

It is noticed that the redrawn images during both reconstruction and prediction periods tend to be more blurred than the original ones. In the least square optimization, the coefficients are determined so that the deviation from the original images is minimized in a statistical sense. As a result, estimated brightness tends to take an intermediate value, and the contrast generally becomes weaker. 


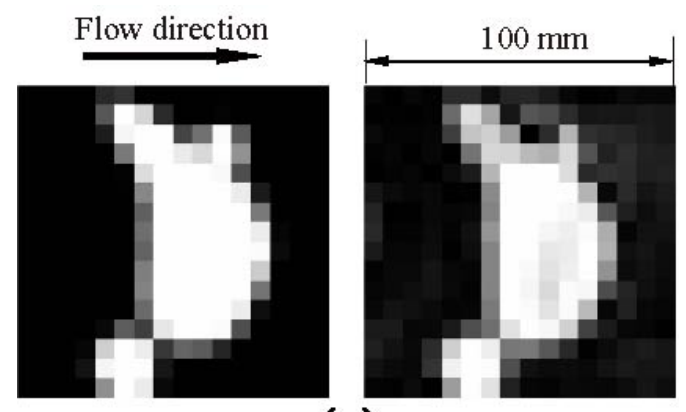

(a)

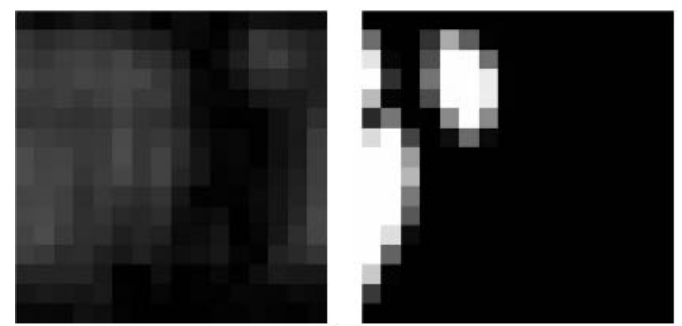

(b)

Figure 6. Comparison between the reconstructed images (left) and original ones (right).

Examples are taken from Case C: (a) high correlation frame $\left(R_{n}=0.97\right.$ over the entire domain); (b) low correlation frame $\left(R_{n}=0.46\right)$.
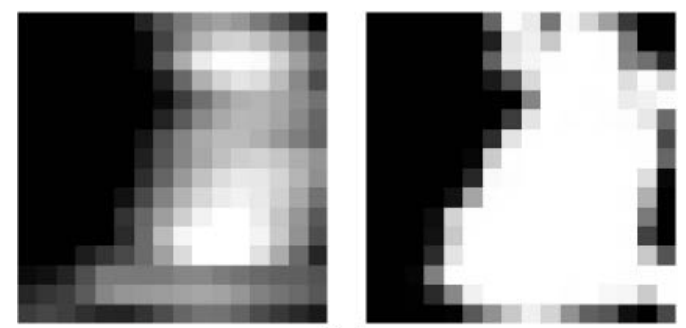

(a)

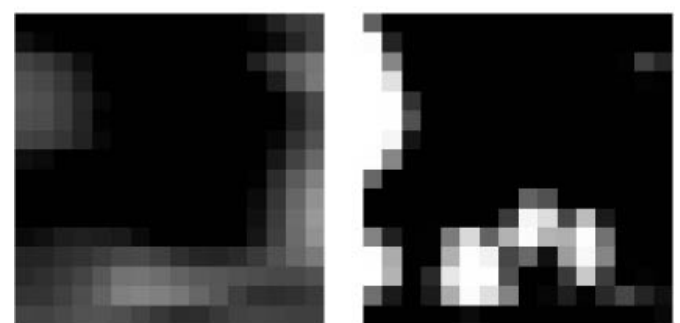

(b)

Figure 7. Comparison between the predicted images (left) and original ones (right) from Case C. Notation is the same as figure 6 . (a) $R_{n}=0.71$; (b) $R_{n}=0.41$ (both over the entire domain).

\subsection{Detectable domain}


It should be remembered that the original image size covers a $100 \mathrm{~mm} \times 100 \mathrm{~mm}$ area in the test section, while the electrode array extends only $45 \mathrm{~mm} \times 45 \mathrm{~mm}$. Hence, the accuracy of the prediction significantly deteriorates outside the array. In fact, such a trend becomes clear by analyzing the time-averaged correlation.

Figure 8 draws the contours of the time-averaged correlation for various flow conditions. We can clearly see that the region covered by the electrodes indicates high correlation. The downstream area also retains higher correlation than the upstream area. This may be due to the position of the counter electrode, which is located downstream. Voltages at the 16 electrodes are influenced not only by the surface phenomena but also by the current paths to the counter electrode. Since the line of sight from the array to the counter electrode is inclined to the downstream direction, the correlation can be retained downstream of the array.

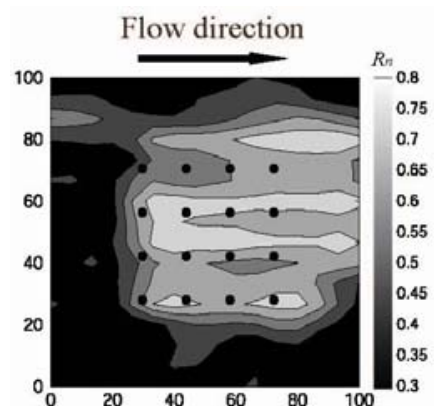

(a)

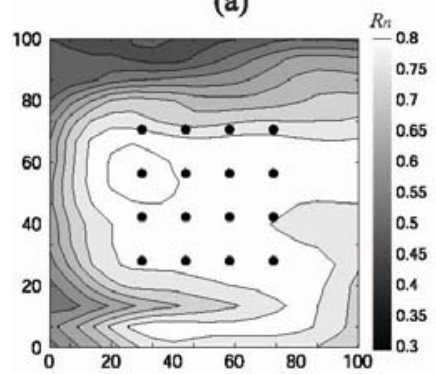

(c)

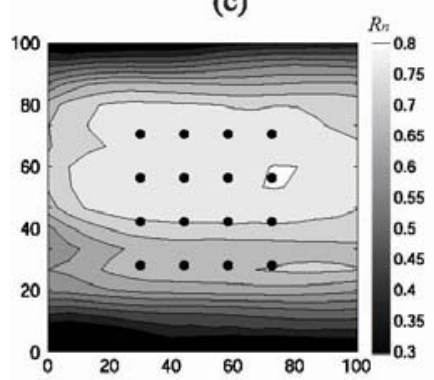

(e)

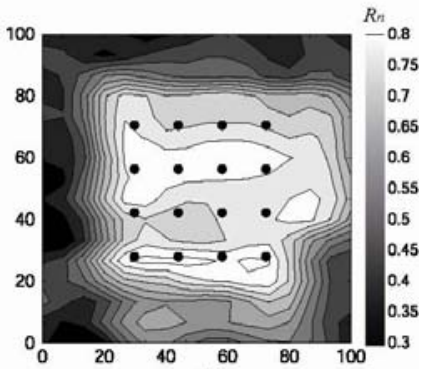

(b)

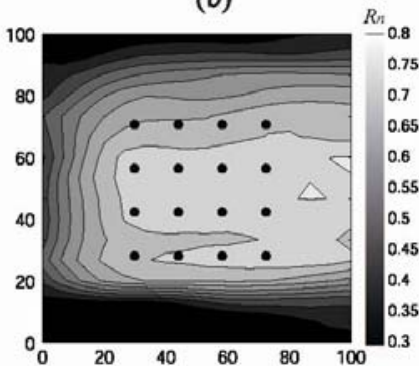

(d)

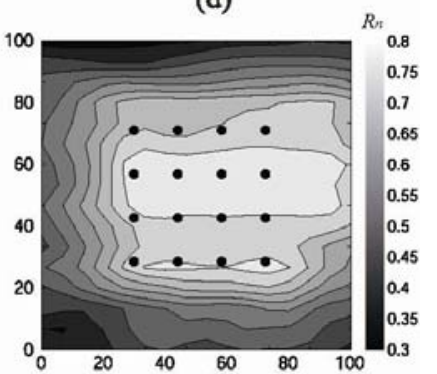

(f)

Figure 8. Contours of time-averaged correlation. Black dots represent the electrodes, and the flow direction is from left to right. (a) Case A; (b) Case B; (c) Case C; (d) Case D; (e) Case E; (f) Average of (a) (e). 
We should note that even if we vary the grids of the images as $8 \times 8,16 \times 16$ and $32 \times 32$ to increase the resolution, the correlation coefficient of Case C only changes as $R=0.864$, 0.841 and 0.819 for the reconstruction and $R=0.728,0.704$ and 0.687 for the prediction, respectively. Thus, we can assure that the image resolution has less impact on the correlation as long as the typical bubble size is resolved.

Figure 9 plots the correlations of the reconstructed and predicted images averaged in both space and time over three different domain sizes at $\alpha=10 \%$ and $20 \%$ (Cases A and C). For all cases, the correlations increase with reducing domain size, which is consistent with the observation above. Even at the lower void fraction $(\alpha=10 \%)$, the correlation during the prediction period is as high as $R=0.455$ for the $50 \mathrm{~mm} \times 50 \mathrm{~mm}$ area. In the following discussion, we evaluate the correlation inside the $50 \mathrm{~mm} \times 50 \mathrm{~mm}$ square.

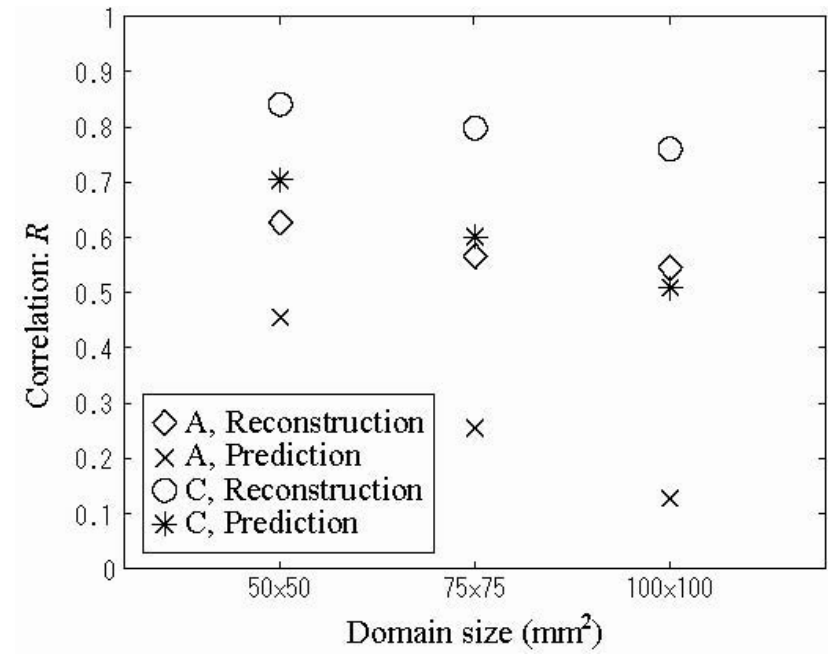

Figure 9. Correlations averaged in space and time for different domain sizes $(U=0.5 \mathrm{~m} / \mathrm{s})$.

Examples are taken from Case $\mathrm{A}(\alpha=10 \%)$ and Case $\mathrm{C}(\alpha=20 \%)$.

\subsection{Dependence on the sampling frame number}

The agreement between the redrawn and original images also depends on the sampling frame number. With varying the sampling number, we perform the Stochastic Estimation to determine the coefficients. Figure 10 compares the correlations averaged over the last 500 frames with coefficients given from three different sampling numbers. The agreement generally becomes better with increasing sampling number. The only exception is Case A ( $\alpha=10 \%$ and $U=0.5 \mathrm{~m} / \mathrm{s}$ ), where the value of correlation indicates that the Stochastic Estimation is nearly invalid for this condition. However, the other four cases exceed $R=0.7$ 
with 2000 sampling frames.

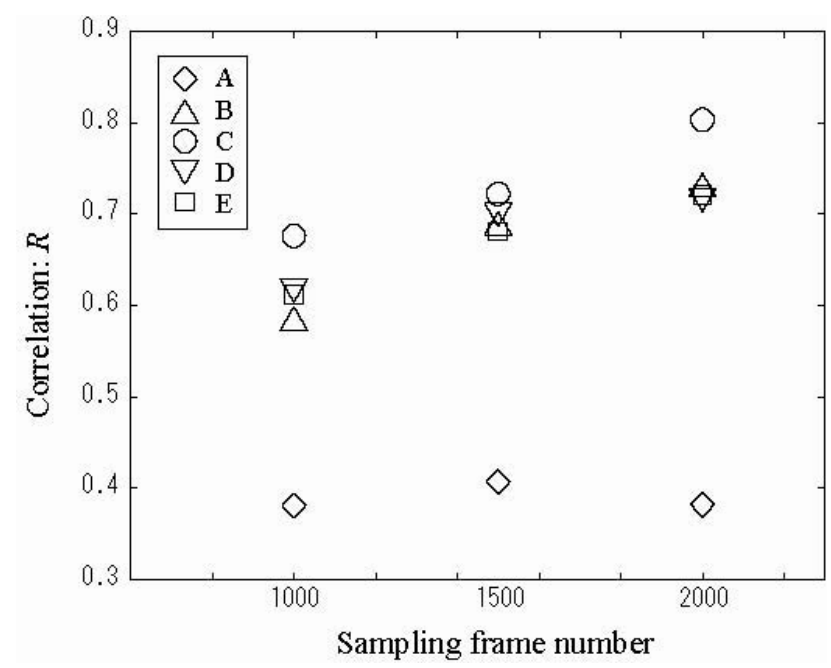

Figure 10. Correlations averaged in space and time over 500 predicted images with different sampling frame numbers. All cases (A - E) are plotted.

It should be noted that the correlation during the reconstruction period shows an opposite trend with the sampling frame number. Figure 11 plots the averaged correlations of the reconstructed images for all cases. It demonstrates that the correlation slightly declines with the sampling number. Because we determine the coefficients based on the least square optimization in the Stochastic Estimation, the reconstruction process becomes overdetermined when the sampling frames are more than the number of the electrodes. The correlations during the reconstruction and prediction periods would eventually become comparable values with sufficient sampling frames.

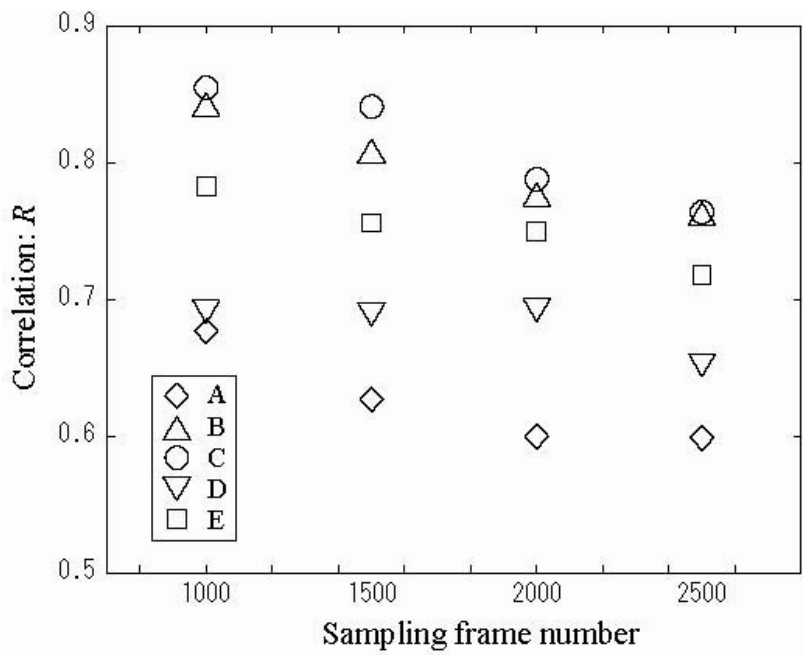

Figure 11. Correlations of reconstructed images averaged in space and time with different sampling frame numbers. All cases (A - E) are plotted. 


\subsection{Dependence on the void fraction and flow velocity}

We summarize the averaged correlations for various void fractions and flow velocities in table

2. The correlations during the prediction period apparently increase with increasing void fraction. All the correlations during the reconstruction period exceed $R=0.6$ with only one exception (Case A). Figure 12 displays an example of reconstructed and predicted images in Case A $(U=0.5 \mathrm{~m} / \mathrm{s}$ and $\alpha=10 \%)$. Compared with figure 6 , the correlation tends to be lower because the sizes of bubbles are smaller at the low void fraction. In particular, the typical bubble size is smaller than the electrode spacing in Case A.

Table 2. Comparison of correlations averaged in time and space for different conditions. The correlations inside the $50 \mathrm{~mm} \times 50 \mathrm{~mm}$ area are listed.

\begin{tabular}{l|cc|cc|cc|c}
\hline & $\begin{array}{c}\text { Reconst. } \\
\text { First 1000 frames }\end{array}$ & $\begin{array}{c}\text { Predict. } \\
\text { Las00 frames }\end{array}$ & $\begin{array}{c}\text { Reconst. } \\
\text { First 1500 frames }\end{array}$ & $\begin{array}{c}\text { Predict. } \\
\text { Last 1000 frames }\end{array}$ & $\begin{array}{c}\text { Reconst. } \\
\text { First 2000 frames }\end{array}$ & $\begin{array}{c}\text { Predict. } \\
\text { Last 500 frames }\end{array}$ & $\begin{array}{c}\text { Reconst. } \\
\text { All 2900 frames }\end{array}$ \\
\hline Case A & 0.677 & 0.364 & 0.627 & 0.455 & 0.600 & 0.382 & 0.584 \\
Case B & 0.841 & 0.575 & 0.807 & 0.647 & 0.775 & 0.725 & 0.767 \\
Case C & 0.855 & 0.622 & 0.841 & 0.704 & 0.788 & 0.803 & 0.776 \\
Case D & 0.693 & 0.536 & 0.691 & 0.592 & 0.694 & 0.717 & 0.668 \\
Case E & 0.783 & 0.553 & 0.756 & 0.560 & 0.750 & 0.721 & 0.724 \\
\hline
\end{tabular}

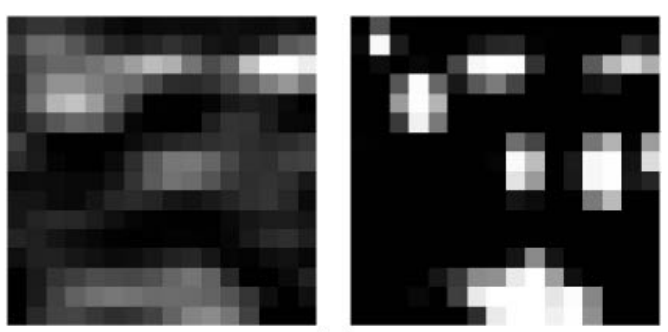

(a)

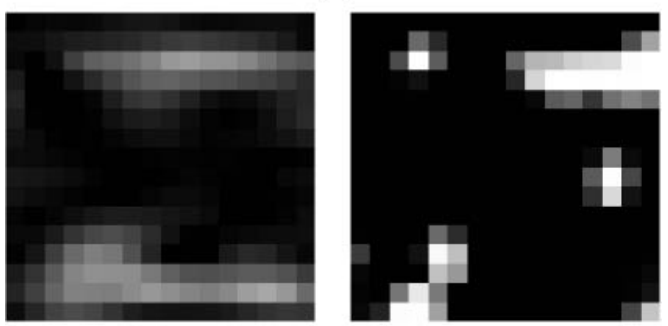

(b)

Figure 12. Comparison between the reconstructed images (left) and original ones (right) from Case A: (a) high correlation frame $\left(R_{n}=0.56\right.$ over the entire domain); (b) low correlation frame $\left(R_{n}=0.20\right)$. 
We plot the averaged correlations for different void fractions in figure 13, where the values are taken from table 2 at $U=0.5 \mathrm{~m} / \mathrm{s}$ with 1500/1000 frames for reconstruction and prediction, respectively. At a constant velocity, the average bubble size becomes larger as the void fraction increases. We confirm that the correlations during both reconstruction and prediction periods increase with increasing bubble size. At the lower void fraction $(\alpha=$ $10 \%$ ), the bubble size is too small to predict the distribution although the reconstructed results are acceptable. This indicates that detectable bubble size is on the order of the sensor spacing. We should additionally note that the numbers of bubbles are different among these three conditions. In general, the correlations are higher with greater numbers of bubbles.

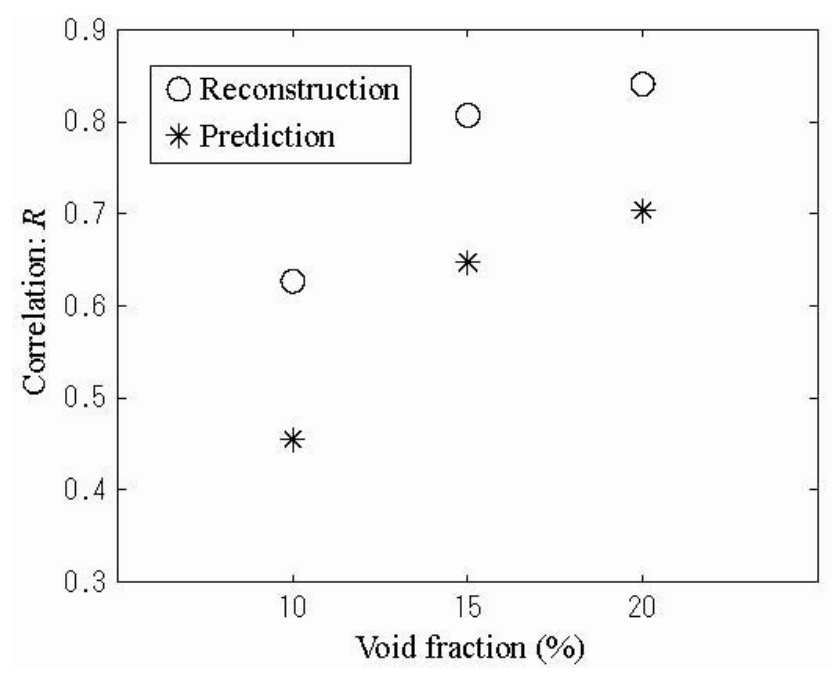

Figure 13. Averaged correlations among different void fractions $(U=0.5 \mathrm{~m} / \mathrm{s})$. The reconstructed periods are averaged over 1500 frames, and the predicted periods over 1000 frames (refer to table 2).

On the other hand, the relation with the flow velocity is not clear. Figure 14 similarly plots the averaged correlation as a function of the velocity at $\alpha=20 \%$ and shows that the correlation is not monotonically decreasing with the velocity. In fact, the projected void fraction is nearly unchanged with the flow velocity (see table 1). The best result corresponds to the lowest velocity, and the other two conditions similarly keep high correlation. Because the sizes of bubbles are nearly uniform at the higher void fraction, the agreement with the actual bubble distribution is relatively high. 


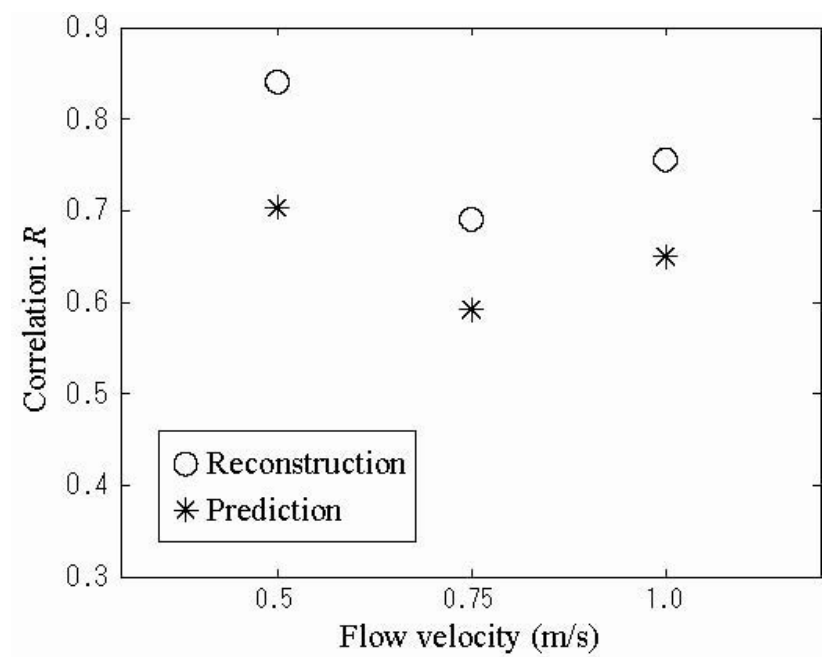

Figure 14. Averaged correlations among different mean velocities $(\alpha=20 \%)$. The numbers of averaged frames are the same as figure 13 (refer to table 2).

\subsection{Off-design conditions processed with a single set of coefficients}

In the preceding sections, we have reconstructed and predicted the bubble distributions based on the coefficients generated from the same condition. To examine the capabilities of the proposed algorithm for off-design conditions, we recalculate coefficients using all the first 1500 frames from each condition (i.e. a total of 7500 frames) and apply this single set of coefficients to all the conditions in the Stochastic Estimation. Figure 15 plots the averaged correlations processed with the single set of coefficients at different void fractions. The corresponding plot in the previous section is figure 13, which is processed with the coefficients for each case. Because the reconstruction period in figure 15 only occupies one fifth of the sampling frames, the correlations of the reconstruction process are lower than those in figure 13. On the other hand, the predictions at higher void fractions $(15 \%$ and 20\%) keep correlations nearly comparable to the previous results. In Case A, however, typical bubble sizes become smaller than the electrode spacing, and the correlation substantially drops, as mentioned before.

The averaged correlations at different velocities are similarly plotted in figure 16 . Compared with figure 14, the correlations during the reconstruction period are as low as those of the prediction period, but all of them are still higher than $R=0.5$. Therefore, it is fair to conclude that the accuracy of reconstruction is significantly deteriorated at off-design conditions; however, the prediction capability can be maintained by processing the sampling 
frames over a wider range of flow conditions although the detectable bubble size still appears to be restricted by the electrode spacing.

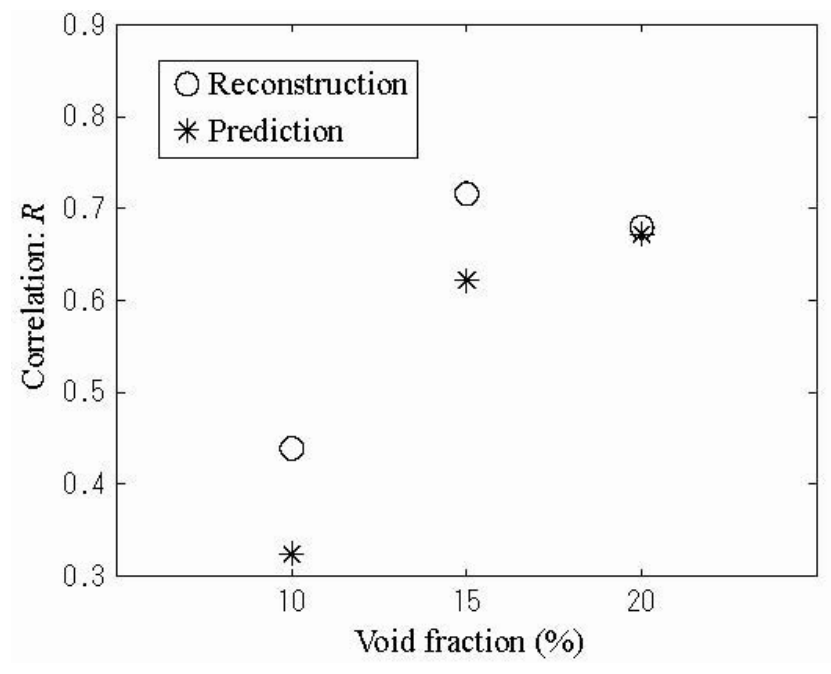

Figure 15. Averaged correlations among different void fractions $(U=0.5 \mathrm{~m} / \mathrm{s})$ processed with a single set of coefficients. The coefficients are calculated over 7500 frames from Cases AE. The numbers of averaged frames for both reconstruction and prediction are the same as figure 13 .

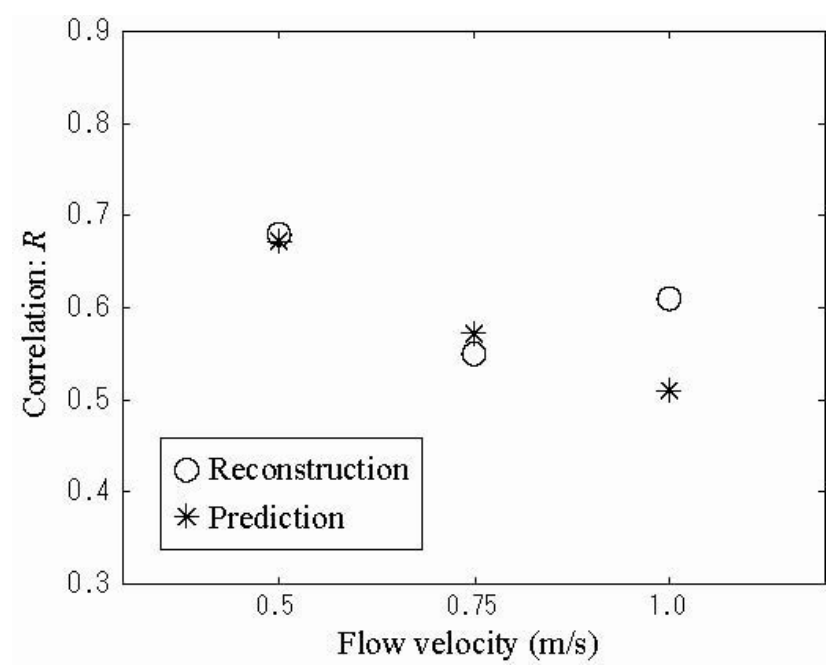

Figure 16. Averaged correlations among different mean velocities $(\alpha=20 \%)$ processed with a single set of coefficients. The numbers of averaged frames and the set of coefficients are the same as figure 15 .

\section{Conclusions}

We have proposed a bubbly-flow reconstruction technique based on the Stochastic Estimation 
and demonstrated it by experiments using a 16 channel electrode array embedded in a horizontal channel. Assuming linear correlation between a local void fraction and electrical impedance, we pre-determine their correlation coefficients with simultaneous image measurement. The reconstruction technique can then estimate instantaneous two-dimensional bubble distribution only from electrical-impedance data over time.

By evaluating the proposed technique, we have found the following features and limitations:

1) The detectable domain of the measuring system has been analyzed. The region covered by the electrode array retains high correlation (as high as $R=0.7$ for most cases); in contrast, the accuracy of the prediction significantly deteriorates outside the array.

2) The sampling frame number must be carefully selected to obtain desirable correlation coefficients. The accuracy of the prediction is generally improved by increasing the sampling frames; however, an opposite trend has been observed during the reconstruction period.

3) We have varied the void fraction at a constant velocity, which accordingly changes the bubble size. The results have shown that the correlation becomes higher as the bubble sizes increase, i.e. at higher void fractions. The electrode spacing appears to restrict the detectable bubble size.

4) By applying a single set of coefficients to a range of void fractions and flow velocities, we have demonstrated that the prediction capability of the Stochastic Estimation can be retained (the averaged correlation is higher than $R=0.5$ ) except for the case when the typical bubble size is smaller than the electrode spacing.

For practical use, we may wish to estimate the distribution of bubbles whose diameter is on the order from a few millimeters to tens of micrometers. This should be technically feasible in a certain degree by clustering smaller electrodes with narrower spacing. An issue of the electrical impedance under such a condition is the durability of electrodes, which must be overcome. Other experimental constraints, such as the size of the array and the number of the sampling frames, can be controlled arbitrarily. If we target smaller bubbles, typically generated using water electrolysis, the measurement should focus on "the distribution of the void fraction" rather than "the distribution of bubbles." Although the algorithm of the 
Stochastic Estimation is still applicable to such conditions in principle, the image processing introduced in this study must be modified so that the distribution of the actual void fraction can be visualized.

There remains another issue of the velocity scaling if we apply the current results for practical use. At typical cruise conditions, the flow velocity is an order of magnitude higher, and this probably enhances the non-uniformity of the bubble shapes and possibly overlapping of bubbles in a two dimensional view. However, as deduced from section 5.5, we can keep the accuracy of the prediction by determining the coefficients based on wide ranges of void fractions and flow velocities. The key is to design an array system that covers wide ranges of flow conditions.

In terms of void-fraction measurement, more sophisticated algorithms, such as impedance tomography, have been introduced for other applications. Those techniques may retain higher spatial resolution for steady conditions, but require careful calibration, computational time for iteration and pairs of an emitter and a detector in the line of sight. In contrast, the proposed approach needs only pre-computed coefficients which include the properties of the measuring system and the flow conditions. Hence, we should calibrate an array in a laboratory test by performing the reconstruction process and apply the set of the same coefficients obtained in the test for the actual operation in principle. In addition, it is convenient to install an electrode array by insulating them from the hull and taking the entire hull as a counter-electrode. Thus, the current technique minimizes computational cost and simplifies the implementation for feedback flow-control applications.

This study is just a first step toward establishing a feedback flow-control system using a bubble-injection method. Many past studies regarding micro-bubble/bubble-injection methods have investigated the relation between the bubble distribution and drag reduction over ranges of void fractions, velocities and other flow parameters. These results provide the guidelines for the target state toward which unsteady bubbly flows should be forced. Therefore, to close the feedback loop in the future study, we must develop the strategy for unsteady bubble injection to maximize drag reduction. Namely, we must investigate the bubbly flow response as a function of air injection. 


\section{REFERENCES}

[1] Madavan N K, Deutsch S and Merkle C L 1985 Measurements of local skin friction in a microbubble-modified turbulent boundary layer J. Fluid Mech. 156 237-256

[2] Guin M M, Kato H, Yamaguchi H, Maeda M and Miyanaga M 1996 Reduction skin friction by microbubble and its relation with near-wall bubble concentration in a channel $J$. Mar. Sci. Technol. 1 241-254

[3] Kato H, Iwashina T, Miyanaga M and Yamaguchi H 1999 Effect of microbubbles on the structure of turbulence in a turbulent boundary layer. J. Mar. Sci. Technol. 4 155-162

[4] Kodama Y, Kakugawa A, Takahashi T and Kawashima H 2000 Experimental study on micro-bubbles and their applicability to ships for skin friction reduction Int. J. Heat Fluid Flow 21 582-588

[5] Xu J, Maxey M R and Karniadakis G E 2002 Numerical simulation of turbulent drag reduction using micro-bubbles J. Fluid Mech. 468 271-281

[6] Deutsch S, Moeny M, Fontaine A A and Petrie H 2004 Microbubble drag reduction in rough walled turbulent boundary layers with comparison against polymer drag reduction Exp. Fluids 37 731-744

[7] Lu J, Fernandez A and Tryggvason G 2005 The effect of bubbles on the wall drag in turbulent channel flow Phys. Fluids 17095102

[8] Murai Y, Fukuda H, Oishi Y, Kodama Y and Yamamoto F 2007 Skin friction reduction by large air bubbles in a horizontal channel flow Int. J. Multiphase Flow 33 147-163

[9] Hess D E and Fu T C 2003 Impact of flow control technologies on naval platforms AIAA paper AIAA-2003-3568

[10] Gillies E A 1998 Low-dimensional control of the circular cylinder wake J. Fluid Mech. 371 157-178

[11] Wambsganss M W, Jendzeczyk J A, France D M and Obot N T 1992 Frictional pressure gradients in two-phase flow in a small horizontal rectangular channel Exp. Thermal Fluid Sci. $540-56$

[12] Fukuda H, Murai Y, Oishi Y, Koukawa M and Yamamoto F 2004 Direct measurement of wall-shear stress and flow visualization of bubbles in a horizontal channel (Fukui: ISAME) 
$253-258$

[13] Glickstein S S, Vance W H and Joo H 1995 Void fraction measurement using neutron radiography Nucl. Sci. Eng. 121 153-161

[14] Cho K H C, Kim S and Lee Y J 2001 Impedance imaging of two-phase flow field with mesh grouping method Nucl. Eng. Des. 204 57-67

[15] Huang Z, Wang B and Li H 2003 Application of electrical capacitance tomography to the void fraction measurement of two-phase flow IEEE Trans. Instrum. Meas. 52 (1) 7-12

[16] Ceccio S L and George D L 1996 A review of electrical impedance techniques for the measurement of multiphase flows Trans. ASME J. Fluid Eng. 118 (2) 391-399

[17] Song C $-\mathrm{H}$, Chung M K and No H C 1998 Measurements of void fraction by an improved multi-channel conductance void meter Nucl. Eng. Des. 184 (2) 269-285

[18] Richter S, Aritomi M, Prasser H-M and Hampel R 2002 Approach towards spatial phase reconstruction in transient bubbly flow using a wire-mesh sensor Int. J. Heat Mass Trans. $1063-1075$

[19] Cho J, Perlin M and Ceccio S L 2005 Measurement of near-wall stratified bubbly flows using electrical impedance Meas. Sci. Tecnol. 16 1021-1029

[20] Fukuda Y, Suzuki T and Yamamoto F 2005 Reconstruction of bubbly flow in a horizontal channel using stochastic estimation. (Pukyong: ISAME) 332-336

[21] Zhou W, Suzuki T and Yamamoto F 2007 Reconstruction of bubbly flow based on shear stress using stochastic estimation (Sapporo: JSMF) 24-25

[22] Adrian R J and Moin P 1988 Stochastic estimation of organized turbulent structure: homogeneous shear flow J. Fluid Mech. 190 531-559

[23] Cole D, Glauser M and Guezennec Y 1991 An application of the stochastic estimation to the jet mixing layer Phys. Fluids 4 (1) 192-194

[24] Murai Y, Oishi Y, Takeda Y and Yamamoto F 2006 Turbulent shear stress profiles in a bubbly channel flow assessed by particle tracking velocimetry Exp. Fluids $\mathbf{4 1}$ 343-352 